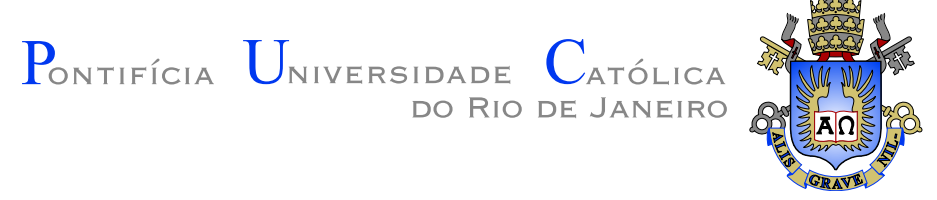

Karen Patricia Guevara Ramos

\title{
Propagação de Incertezas via Expansão por Caos Polinomial em Simulação de Reservatórios de Petróleo
}

Dissertação apresentada ao Programa de Pos-graduação em Engenharia Elétrica da PUC-Rio como requisito parcial para obtenção do ttulo de Mestre em Engenharia Elétrica.

Orientador: Prof. Marco Aurélio Cavalcanti Pacheco 


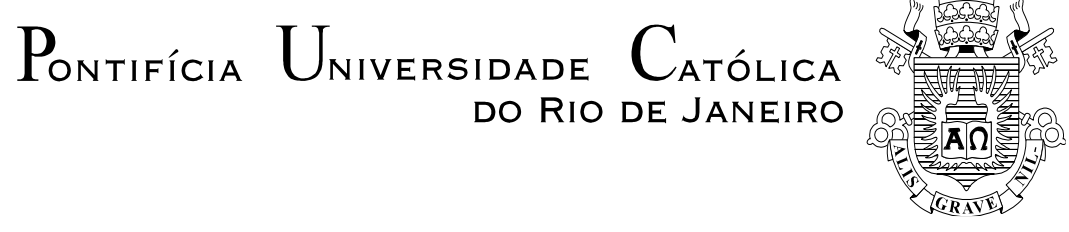

Karen Patricia Guevara Ramos

\begin{abstract}
Propagação de Incertezas via Expansão por Caos Polinomial em Simulação de Reservatórios de Petróleo
\end{abstract}

Dissertação apresentada como requisito parcial para obtenção do grau de Mestre pelo Programa de Pós-Graduação em Engenharia Elétrica do Departamento de Engenharia Elétrica do Centro Técnico Científico da PUC-Rio. Aprovada pela Comissão Examinadora abaixo assinada.

Prof. Marco Aurélio Cavalcanti Pacheco

Orientador

Departamento de Engenharia Elétrica - PUC-Rio

Prof. Carlos Kubrusly

Departamento de Engenharia Elétrica - PUC-Rio

Dr. Alexandre Anozé Emerick

Petróleo Brasileiro - Rio de Janeiro - Matriz

Prof. Douglas Mota Dias

Departamento de Engenharia Elétrica - PUC-Rio

Prof. José Eugenio Leal

Coordenador Setorial do Centro

Técnico Científico

Rio de Janeiro, 13 de março de 2014 
Todos os direitos reservados. É proibida a reprodução total ou parcial do trabalho sem autorização da universidade, da autora e do orientador.

\section{Karen Patricia Guevara Ramos}

Graduou-se em Ciências Atuariais pela Universidade de Costa Rica (UCR) em 2010.

Ficha Catalográfica

Ramos, Karen Patricia Guevara

Propagação de incertezas via expansão por caos polinomial na simulação de reservatórios de petróleo / Karen Patricia Guevara Ramos; orientador: Marco Aurélio Cavalcanti Pacheco. -2014.

113 f.: il. (color.) ; $30 \mathrm{~cm}$

Dissertação (mestrado)- Pontifícia Universidade Católica do Rio de Janeiro, Departamento de Engenharia Elétrica, 2014.

Inclui bibliografía.

1. Engenharia Elétrica - Teses. 2. Caos polinomial; . 3. propagação de incertezas; . 4. simulação de reservatórios; 5. redução do custo computacional; . 6. polinômios ortogonais.. I. Pacheco, Marco Aurélio Cavalcanti. II. Pontifícia Universidade Católica do Rio de Janeiro. Departamento de Engenharia Elétrica. III. Título. 


\section{Agradecimentos}

A Deus por tudo, em particular por me ajudar nos momentos que mais precisava.

Ao meu orientador Prof. Marco Aurélio, pelo apoio e oportunidade de ser parte da equipe do Laboratório ICA.

Aos meus pais, Carolina e Santos, pela educação, carinho e apoio em cada etapa da minha vida e que, mesmo distante, sempre se mostraram presentes.

Ao meu namorado Liev, por toda sua ajuda incondicional desde o início desta etapa, mas, sobretudo, pela paciência que sempre teve comigo nos meus momentos de irritação e desânimo.

Aos meus irmãos e segunda família Ferreira Maribondo; que de uma forma ou de outra me estimularam e me ajudaram.

A João, por seu apoio incondicional nos momentos bons e não tanto, e por acreditar sempre em mim. Também aos meus colegas de equipe Iuri e Pedro, pelos ensinamentos e disposição em me ajudar neste trabalho.

Aos professores Kubrusly (PUC) e William, Alexander e Roldán (UCR) que sempre me incentivaram e me brindaram todo seu apoio.

Aos professores que participaram da Comissão examinadora.

A CAPES, à PUC-Rio, e à Petrobrás, pelos auxílios concedidos, sem os quais este trabalho não poderia ter sido realizado. 


\section{Resumo}

Ramos, Karen Patricia Guevara; Pacheco, Marco Aurélio Cavalcanti. Propagação de Incertezas via Expansão por Caos Polinomial em Simulação de Reservatórios de Petróleo. Rio de Janeiro, 2014. 113 p. Dissertação de Mestrado - Departamento de Engenharia Elétrica, Pontifícia Universidade Católica do Rio de Janeiro.

Este trabalho tem por objetivo investigar a redução do custo computacional associado ao cálculo das principais estatísticas das saídas dos modelos de propagação de incertezas. Para tal, apresentamos uma implementação alternativa ao método tradicional de Monte Carlo, chamado Caos Polinomial; que é adequado a problemas onde o número de variáveis de incerteza não é muito alto. No método Caos Polinomial, o valor esperado e a variância das saídas do simulador são diretamente estimados, como funções de distribuições de probabilidade de variáveis de incerteza na entrada do simulador. A principal vantagem do método de Caos Polinomial é que o número de pontos necessários para uma boa estimativa das estatísticas da saída de um simulador, comparado com Monte Carlo, é menor. Aplicações de Caos Polinomial em reservatórios de petróleo serão apresentadas para a propagação de até quatro variáveis, apesar do método poder ser aplicado a problemas de dimensões maiores. Nossos principais resultados são aplicados a dois modelos de reservatórios de petróleo sintéticos.

\section{Palavras-chave}

Caos polinomial; propagação de incertezas; simulação de reservatórios; redução do custo computacional; polinômios ortogonais. 


\section{Abstract}

Ramos, Karen Patricia Guevara; Pacheco, Marco Aurélio Cavalcanti (Advisor). Uncertainty Propagation using Polynomial Chaos Expansion in Oil Reservoir Models. Rio de Janeiro, 2014. 114p. MSc. Dissertation - Departamento de Engenharia Elétrica, Pontifícia Universidade Católica do Rio de Janeiro.

In this work we investigate the reduction of the computational cost of the calculus of statistical moments of simulator's output in uncertainties propagation's models. For do that, we present an alternative's implementation to the traditional Monte Carlo's Method, called Polynomial Chaos; that is adequate to problems where the number of uncertain variables is not so high. In the Polynomial Chaos method, the expectation and the variance of the simulator's output are directly estimated, as functions of the probability distribuition of the uncertain variables in simulator input. The great advantage of Polynomial Chaos is that number of points necessary for a good estimation of the output statistics have smaller magnitude, compared to the Monte Carlo Method. Applications of Polynomial Chaos on oil reservoir simulations will be presented. As it is just a preliminar implementation, we just treat propagation's problems with at most four uncertainties variables, despite of the method being applicable to problems with more dimensions. Our main results are applied to two models of synthetic oil reservoirs.

\section{Keywords}

Polynomial chaos; propagation of uncertainties; reservoir simulation; reduction of computational cost; orthogonal polynomials. 


\section{Sumário}

$\begin{array}{lll}1 & \text { Introdução } & \mathbf{1 0}\end{array}$

2 Conceitos de Teoria da Probabilidade $\quad \mathbf{1 6}$

2.1 Espaços de Probabilidade $\quad 16$

2.2 Funções Mensuráveis 18

2.3 Operador Integração 23

2.4 Espaço $L^{2} \quad 25$

3 Polinômios Ortogonais e Quadratura Gaussiana 29

3.1 Polinômios Ortogonais 30

3.2 Polinômios Ortogonais Clássicos 35

$\begin{array}{ll}\text { 3.3 Quadraturas Gaussianas } & 40\end{array}$

3.4 Alguns exemplos de quadraturas Gaussianas 44

$4 \quad$ Expansão de Caos Polinomial $\quad 51$

4.1 Caos Polinomial Homogêneo 52

4.2 Caos Polinomial Generalizado 63

$5 \quad$ Propagação de Incertezas $\quad \mathbf{6 7}$

5.1 Formulação Matemática 68

5.2 Método Monte Carlo 70

5.3 Método da Quadratura $\quad 72$

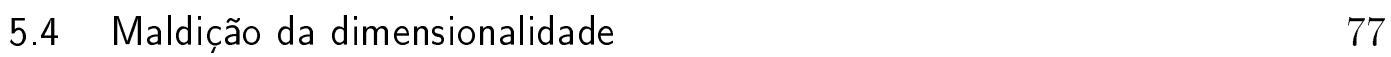

6 Estudo de Caso $\quad 81$

6.1 Modelagem $\quad 81$

6.2 Estudo de Caso 1

6.3 Estudo de Caso $2 \quad 86$

6.4 Problema da Maldição da Dimensionalidade 96

$\begin{array}{lll}7 & \text { Conclusões e Trabalhos Futuros } & 98\end{array}$

8 Referências Bibliográficas $\quad 101$

9 Apêndice - Espaços de Hilbert $\quad 107$

$\begin{array}{ll}9.1 \text { Ortogonalidade } & 109\end{array}$

9.2 Bases Ortonormais 109

9.3 Processo de Ortogonalização de Gram- Schmidt 111

9.4 Teorema das Series de Fourier 113 


\section{Lista de figuras}

Figura 2.1 Densidade de Probabilidade Lognormal 21

Figura 3.1 Polinômios de Legendre 36

$\begin{array}{lll}\text { Figura 3.2 Polinômios de Laguerre } & 37\end{array}$

Figura 3.3 Polinômios de Hermite probabilísticos $\quad 39$

$\begin{array}{lll}\text { Figura } 3.4 & \text { Esquema Askey }\end{array}$

Figura 4.1 Polinômios de Hermite Ordem 0, 1, 2. 60

Figura 4.2 Polinômios de Hermite Ordem 3. 62

$\begin{array}{lll}\text { Figura 5.1 Propagação de Incertezas } & 70\end{array}$

Figura 5.2 Conjunto de amostras aleatórias no quadrado unitário $\quad 79$

Figura 6.1 Campo Tutorial $\quad 83$

Figura 6.2 Estudo Paramétrico: VPL vs POR 84

Figura 6.3 Estudo Paramétrico: VPL vs PERMI 84

Figura 6.4 Campo PUNQ-S3 86

Figura 6.5 Estudo Paramétrico: Óleo Acumulado vs Porosidade 87

Figura 6.6 Estudo Paramétrico: Óleo Acumulado vs Permeabilidade i 88

Figura 6.7 Estudo Paramétrico: Óleo Acumulado vs Permeabilidade j 88

Figura 6.8 Estudo Paramétrico: Óleo Acumulado vs Permeabilidade k 88 


\section{Lista de tabelas}

Tabela 3.1 Zeros e pesos para quadratura de Gauss-Legendre 46

Tabela 3.2 Zeros e pesos para quadratura de Gauss-Laguerre 47

Tabela 3.3 Zeros e pesos para quadratura de Gauss-Hermite Físicos 48

Tabela 3.4 Zeros e pesos para quadratura de Gauss-Hermite Probabilísticos 49

Tabela 4.1 Exemplo do multi-índice de dimensão 4

Tabela 4.2 Polinômios de Hermite de Duas Dimensões 59

Tabela 4.3 Polinômios de Hermite de Três Dimensões 61

Tabela 4.4 Dependência do número de termos com $N$ e $p$. 63

Tabela 4.5 Tabela Askey 65

Tabela 6.1 Momentos do VPL via Monte Carlo 85

Tabela 6.2 Momentos do VPL via Quadratura 85

Tabela 6.3 Monte Carlo - Porosidade $\quad 89$

Tabela 6.4 Quadratura - Porosidade $\quad 90$

Tabela 6.5 Sobol - Porosidade 90

Tabela 6.6 Monte Carlo - Permeabilidade i 91

Tabela 6.7 Quadratura - Permeabilidade i $\quad 91$

Tabela 6.8 Sobol - Permeabilidade i 91

Tabela 6.9 Monte Carlo - Permeabilidade j 92

Tabela 6.10 Quadratura - Permeabilidade j 92

Tabela 6.11 Sobol - Permeabilidade j 92

Tabela 6.12 Amostras - Permeabilidade k $\quad 92$

Tabela 6.13 Quadratura - Permeabilidade k $\quad 92$

Tabela 6.14 Sobol - Permeabilidade k 93

Tabela 6.15 Monte Carlo - Permeabilidade i,j 93

Tabela 6.16 Quadratura - Permeabilidade i,j 93

Tabela 6.17 Sobol - Permeabilidade i,j 94

Tabela 6.18 Monte Carlo - Permeabilidade i,j,k 94

Tabela 6.19 Quadratura - Permeabilidade i,j,k 95

Tabela 6.20 Sobol - Permeabilidade i,j,k 95

Tabela 6.21 Monte Carlo - Porosidade, Permeabilidade i,j,k 95

Tabela 6.22 Quadratura - Porosidade, Permeabilidade i,j,k 96

Tabela 6.23 Sobol - Porosidade, Permeabilidade i,j,k 96

Tabela 6.24 Caso teste $1 \quad 97$ 